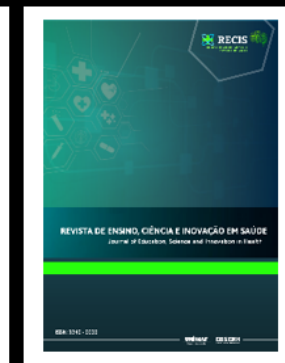

\title{
Avaliação clínica e radiológica dos pacientes portadores de Síndrome Respiratória Aguda Grave e Covid-19 admitidos em um hospital terciário do Vale do São Francisco
}

\section{Clinical and radiological evaluation of patients with Severe Acute Respiratory Syndrome and Covid-19 admitted in a tertiary hospital at the Valley of São Francisco}

\author{
Rebecca Leão Feitoza de Brito ${ }^{1}$, Amanda Thaysa de Oliveira Cruz ${ }^{2}$, Larissa Cavalcante \\ Bomfim $^{2}$, Marcos Duarte Guimarães ${ }^{3}$
}

${ }^{1}$ Discente da Universidade Federal do Vale do São Francisco, ${ }^{2}$ Médico Residente do Hospital Regional de Juazeiro da Bahia, ${ }^{3}$ Docente do Colegiado de Medicina da Universidade Federal do Vale do São Francisco.

Autor correspondente: rebeccalfbrito13@gmail.com

Artigo recebido em 05/11/2020 e aceito em 14/12/2020

\begin{abstract}
RESUMO
As síndromes respiratórias agudas graves são responsáveis por um número considerável de internamentos em Unidades de Terapias Intensiva. Atualmente, este contingente vem crescendo consideravelmente em virtude da pandemia causada pelo novo corona vírus, o SARS-CoV-2. Diferentes aspectos clínicos e radiológicos têm sido relacionados ao desenvolvimento da doença. Este estudo tem como objetivo avaliar aspectos clínicos e radiológicos dos indivíduos admitidos em um hospital regional localizado na região do semiárido do nordeste brasileiro. Foram estudados 104 pacientes de forma retrospectiva, dos quais, 17 (27\%) tiveram testes laboratoriais positivos para Covid-19. Sintomas como dispneia, febre e tosse foram os mais frequentes, presentes em 77,88\%, 72,11\% e 59,61\% dos casos, respectivamente. Idade inferior a 60 anos, sexo masculino, hipertensão, riscos cardiovasculares e diabetes mellitus foram as variáveis clínicas mais frequentes presentes em $66,4 \%, 62 \%, 18,26 \%$ e $10,57 \%$ dos casos, respectivamente. Oitenta e cinco $(81,73 \%)$ pacientes realizaram exame tomográfico do tórax, sendo os achados considerados típicos os mais comuns nos pacientes que testaram positivos para Covid-19, presentes em 70,5\% dos casos. O presente estudo demonstra que a maioria dos achados clínicos e radiológicos foi semelhante a outros estudos da literatura.
\end{abstract}

Palavras-chave: COVID-19; Síndrome Respiratória Aguda Grave; radiologia; diagnóstico; epidemiologia.

\begin{abstract}
Severe acute respiratory diseases are responsible for a considerable number of Intense Care Unit (ICU). Nowadays, this contingent has been growing remarkably due to the new coronavirus, SARS-CoV-2. Different clinical and radiological aspects have been related to the development of this disease. This study aims to evaluate clinical and radiological aspects of individuals admitted to a regional health center at the semi-arid region of northeastern Brazil. 104 patients were retrospectively studied, of which 17 (27\%) had positive laboratory tests for Covid-19. Dyspnea, fever and cough were the most frequent symptoms, present in $77.88 \%, 72.11 \%$ and $59.61 \%$ of cases. Age under 60 years, male, hypertension, cardiovascular risks and diabetes mellitus were the most frequent clinical variables present in $66.4 \%, 62 \%, 18.26 \%$ and $10.57 \%$ cases, respectively. Eighty-five (81.73\%) patients underwent a chest tomography, with typical findings being the most common in patients who tested positive for Covid19, present in $70.5 \%$ of cases. Except for the age group, the data from the present study has been consistent with other studies published in literature.
\end{abstract}

Keywords: COVID-19; Severe Acute Respiratory Syndrome; radiology; diagnosis; epidemiology. 


\section{INTRODUÇÃO}

Pertencente à família Coronaviridae, o SARS-CoV-2 é reconhecido como o agente causador da COVID 19, a qual é responsável pela atual pandemia global. Essa doença é uma das causas da Síndrome Respiratória Aguda Grave (SRAG) e teve início em dezembro de 2019 na cidade de Wuhan, na China. ${ }^{1}$

Este vírus foi sequenciado, e posteriormente identificado como um novo vírus envelopado de RNA de fita simples, descrito como um betacoronavírus. É o terceiro coronavírus emergente em duas décadas, apresentando alto poder de disseminação. ${ }^{2} \mathrm{O}$ que pode justificar essa propagação internacional da doença é a maneira de transmissão, susceptibilidade do hospedeiro e a infectividade do vírus. ${ }^{3}$

As taxas de infecções e óbitos pelas SRAG principalmente causadas pelo SARS-CoV-2 vêm aumentando significativamente em todo globo terrestre. ${ }^{4}$ No Brasil, dados do Ministério da Saúde (MS) demonstraram mais de 170.000 óbitos e 6 milhões de infectados, até o último trimestre de 2020. Nos estados do Nordeste os números também são alarmantes. No mesmo período a Bahia apresentou mais de 400.000 mil casos e 8 mil mortes causadas por esta doença. ${ }^{4}$

Sua apresentação clínica é diversificada, podendo causar sintomas iniciais como febre, tosse seca, dispneia, além de mialgia ou fadiga. Os casos avançados podem evoluir com redução dos níveis de saturação de oxigênio sanguíneo, acometimento pulmonar, distúrbios nos rins e fígado, linfopenia e choque. Estudos também demonstram que pacientes idosos e portadores de comorbidades como doenças crônico-degenerativas são mais susceptíveis ao desenvolvimento da forma grave da doença com aumento das taxas de mortalidade nestes subgrupos populacionais. 5,6

A confirmação diagnóstica da infecção pelo SARS-CoV-2 se dá pela detecção do RNA mensageiro viral através de reação em cadeia da polimerase com transcrição reversa em tempo real (RT-PCR). Testes sorológicos e o exame de tomográfico do tórax também têm sido empregados no diagnóstico dessa doença, por vezes com resultados divergentes. ${ }^{7}$

Em um estudo asiático foi observado que mais de $80 \%$ dos pacientes classificados como graves demonstraram anormalidades nos exames tomográficos do tórax, sendo as opacidades com atenuação em fosco e consolidações periféricas as mais comuns. ${ }^{89,10}$
Com a evolução do quadro, as lesões tendem a se estender pelo parênquima do pulmão, com configurações multifocais bilaterais, predominantemente periférico e subpleural. A depender do tempo de evolução, o modelo padrão pode se apresentar de maneira mista, incluindo outros tipos de opacidades como as reticulares e centrolobulares. ${ }^{11}$

O presente estudo teve o objetivo de analisar aspectos clínicos e radiológicos de pacientes portadores de SRAG e Covid-19 admitidos no Hospital Regional de Juazeiro/BA (HRJ/BA), centro de alta complexidade e referência para atendimento destes pacientes na região do submédio do Vale do São Francisco.

\section{MATERIAL E MÉTODOS}

Foi desenvolvido um estudo retrospectivo transversal observacional em 104 pacientes admitidos no HRJ/BA com quadro de SRAG ou suspeita de infecção pelo Covid-19. Foram coletados dados demográficos como sexo e idade e variáveis clínicas como sinais, sintomas, comorbidades e evolução dos prontuários eletrônicos. A confirmação diagnóstica de covid19 foi obtida através dos testes PCR e sorológicos, quando disponíveis. Foram coletados também os achados radiológicos encontrados nos exames de tomografia computadorizada (TC) destes pacientes que foram classificados quanto a: 1- achados típicos de Covid-19 (vidro fosco peribroncovascular, distribuição periférica e/ou bilateral, pavimentação em mosaico e focos de consolidação) 2- indeterminados ( opacidades em vidro fosco e consolidações de distribuição central, não arredondados, unilaterais) e 3- achados atípicos (consolidação lobar, nódulos centrolobulares, bronquiectasias, escavação pulmonar ou outros achados não classificados com típico ou indeterminado). Os pacientes foram classificados quanto a fase da doença de acordo com o início dos sintomas: 1- precoce, se admitido com até 5 dias do início dos sintomas, intermediário se admitido entre $6^{\circ}$ e $10^{\circ}$ dia de sintomas, fase tardia a partir do $11^{\circ}$ dia do início dos sintomas. Os dados coletados foram analisados através do software SPSS 20.0. O projeto foi aprovado pelo Comitê de Ética em Pesquisa (CEP) com número do CAAE: 33972620.4.0000.8052. 


\section{RESULTADOS}

Dos 104 pacientes admitidos 64 (62\%) eram do sexo masculino e 40 (38\%) do sexo feminino. Cinquenta e sete apresentaram fatores de risco para a infecção respiratória, sendo que $2 / 3$ $(66,4 \%)$ tinham idade inferior a 60 anos, conforme demonstrado na Tabela 01.

Tabela 01 - Fatores de risco mais frequentes nos casos de SRAG

\begin{tabular}{llc}
\hline \multicolumn{1}{c}{ Fatores de risco } & $\mathbf{N}(\%)$ \\
\hline 1. Idade < 60 anos & $69(66,4)$ \\
2. Hipertensão & $19(18,3)$ \\
3. Diabetes Mellitus & $11(10,6)$ \\
4. Pneumopatias crônicas & $7(6,7)$ \\
5. Doença neurológica & $6(5,8)$ \\
crônica & $15(14,4)$ \\
6. Outros*
\end{tabular}

*Outros: Imunodeficiência, obesidade, doença cardiovascular crônica, doença renal crônica, tuberculose, asma, sequela AVC e doença hematológica. Observação: Alguns pacientes possuem mais de um fator de risco.

Os sintomas avaliados demostraram que dispneia, febre e tosse foram os mais frequentes, presentes em $81(77,9 \%), 75(72,1 \%)$ e $62(59,6 \%)$ pacientes, respectivamente (Tabela 02 ).

A saturação baixa de oxigênio $\left(\mathrm{O}_{2}<95 \%\right)$ esteve presente em $24(23,1 \%)$ pacientes. Dos pacientes admitidos com quadro de SRAG, 63 $(60,6 \%)$ fizeram exames laboratoriais no hospital. Dezessete (27\%) tiveram resultados positivos para Covid-19 e 40 (63,5\%), negativos. Enquanto que em $6(9,5 \%)$ paciente os resultados não foram disponibilizados (tabela 03).

Oitenta e cinco $(81,73 \%)$ pacientes realizaram exame tomográfico do tórax. A distribuição quanto a fase da doença no momento do exame: se precoce, intermediário ou tardio correspondeu a: 19 (22\%), 23 (27\%) e 43 (51\%), respectivamente (Tabela 04).

Quanto à classificação dos achados tomográficos houve comparação com os resultados dos 63 pacientes que realizaram testes de diagnóstico para Covid-19.

A distribuição dos achados nos 17 pacientes que testaram positivo foi: $2(11,8 \%)$ pacientes com TC normal, 12 (70,5\%) com achados típicos e $3(17,7 \%)$ com achados atípicos.
A distribuição nos 40 pacientes que testaram negativo foi: 4 (10\%) pacientes com TC normal, $3(7,5 \%)$ com achados típicos, 8 (20\%) com achados indeterminados e $25(62,5 \%)$ com achados atípicos na TC de tórax.

Tabela 02 - Sintomas mais frequentes na admissão dos pacientes do estudo

\begin{tabular}{lc}
\hline \multicolumn{1}{c}{ Sintoma } & $\mathbf{N}(\%)$ \\
\hline 1. Dispneia & $81(77,9)$ \\
2. Febre & $75(72,1)$ \\
3. Tosse & $62(59,6)$ \\
4. Artralgia/Mialgia & $20(19,2)$ \\
5. Cefaleia & $17(16,3)$ \\
6. Dor de garganta & $14(13,5)$ \\
7. Taquipneia & $13(12,5)$ \\
8. Anosmia & $9(8,6)$ \\
9. Astenia & $9(8,6)$ \\
10. Outros* & $44(42,3)$
\end{tabular}

*Outros: Dor torácica, rinorreia, diarreia, congestão nasal, dor abdominal, vômito, convulsão, náuseas, adinamia, sonolência, coriza, inapetência, sudorese, hipotensão e falta de apetite.

Tabela 03 - Resultados dos exames dos pacientes avaliados para a COVID 19

\begin{tabular}{lc}
\hline \multicolumn{1}{c}{ Resultados } & $\mathbf{N}(\boldsymbol{\%})$ \\
\hline 1. Negativos & $40(63)$ \\
2. Positivos & $17(27,0)$ \\
3. Sem Resultado & $6(9,5)$ \\
Total & $63(100)$ \\
\hline
\end{tabular}

Tabela 04 - Quantidade de exames tomográficos de tórax realizados, com a percentagem segundo a fase da doença desde o início dos sintomas

Fase da doença $\mathbf{N}(\%)$
1. Precoce (0 a 5 dias) $19(22)$
2. Intermediária (6 a 10 dias) $23(27)$
3. Tardia (> 10 dias)
$43(51)$ 
Em relação à conduta após admissão, 14 $(13,5 \%)$ pacientes foram admitidos em UTI, 37 $(35,6 \%)$ em enfermaria, e $53 \quad(50,9 \%)$ encaminhados para acompanhamento domiciliar. (Tabela 05).

Tabela 05 - Distribuição das condutas dos pacientes admitidos com SRAG

\begin{tabular}{|c|c|}
\hline Conduta & $\mathbf{N}(\%)$ \\
\hline 1. Internação em UTI & $14(23)$ \\
\hline 2. Internação em enfermaria & $37(62)$ \\
\hline $\begin{array}{l}\text { 3. Acompanhamento } \\
\text { domiciliar }\end{array}$ & $53(15)$ \\
\hline Total & $104(100)$ \\
\hline
\end{tabular}

\section{DISCUSSÃO}

Esta pesquisa destaca os perfis clínicos e tomográficos dos pacientes admitidos em um hospital de referência para admissão de pacientes portadores de SRAG e suspeitos de infecção pelo COVID-19 na região do submédio do Vale do São Francisco.

Pacientes do sexo masculino, com idade inferior a 60 anos, hipertensos, diabéticos e pneumopatas crônicos foram os mais encontrados na presente casuística. Excetuando a faixa etária, os resultados referentes aos aspectos clínicos do presente estudo foram condizentes com a maioria das publicações. ${ }^{12} \mathrm{O}$ fato de encontrarmos nesta casuística uma média de idade inferior à média de idade em outros estudos da literatura, pode ser atribuído ao HRJ/BA ser um hospital de porta aberta, sendo referência para atendimento de pacientes portadores de sintomas respiratórios leves, moderados e graves durante a pandemia.

Observa-se também uma alta frequência de exames tomográficos do tórax no presente estudo, em quantidade semelhante ou superior a outros estudos. Mesmo em pacientes classificados como fase precoce, houve a realização deste exame, provavelmente em virtude da baixa disponibilidade dos testes laboratoriais de diagnóstico do Covid19. Sabe-se também que a TC está presente de forma universal em diversos centros de saúde de pequeno e médio porte. A TC de tórax é um exame de fácil execução, podendo ser realizado em poucos segundos e sem a necessidade do uso de contraste iodado intravenoso. Pode ser indicada como ferramenta alternativa para auxiliar no diagnóstico, classificação dos achados e orientação da conduta daqueles pacientes portadores de SRAG ou suspeitos de infecção do Covid-19. ${ }^{13}$ O período de experiência adquirido no início da pandemia quanto aos achados radiológicos dos exames tomográficos de tórax pode ter sido causa de discordância com os resultados dos demais exames laboratoriais. Por outro lado, sabe-se que o exame de RT-PCR tem seu ápice de desempenho no início dos sintomas, além da possibilidade de exames sorológicos e testes rápidos poderem demonstrar resultados falso-positivos e falso-negativos. Isso leva aos autores reconhecerem as limitações durante a obtenção dos dados. ${ }^{14}$

Opacidades em vidro fosco, consolidações, aspecto arredondado, distribuição periférica e bilateral, foram os achados radiológicos mais frequentes nos pacientes com resultado positivo para o Covid-19, semelhante a outros estudos que fizeram a mesma avaliação. ${ }^{15,16}$

\section{CONCLUSÃO}

As características clínicas e radiológicas do Covid - 19 em uma região do semiárido nordestino brasileiro foram condizentes com dados da literatura. Estas informações são relevantes para futuras tomadas de decisões no âmbito institucional bem como para adoção de medidas de saúde pública que minimizem os impactos desta doença na população local.

\section{AGRADECIMENTOS}

A pesquisa recebeu apoio da coordenação do Hospital Regional de Juazeiro da Bahia, instituição que abrange o submédio São Francisco, local onde aconteceu a coleta dos dados.

\section{REFERÊNCIAS}

1. Munster VJ, Koopmans M, van Doremalen $\mathrm{N}$, et al. A novel coronavirus emerging in china - key questions for impact assessment. N Eng J Med. 2020; 382:6924. https://doi.org/10.1056/NEJMp2000929

2. Zhu N, Zhang D, Wang W, et al. A Novel Coronavirus from Patients with Pneumonia in China, 2019. N Engl J Med. 2020;382(8):727-733. https://doi.org/10.1056/NEJMoa2001017

3. Anderson RM, Heesterbeek H, Klinkenberg D, et al. How will countrybased mitigation measures influence the course of the COVID-19 epidemic? Lancet. 2020; 395(10228):931- 
934. $\quad$ https://doi.org/10.1016/S0140$\underline{6736(20) 30567-5}$

4. Ministério da saúde. [on line]. Brasília, Brasil; 2020. [capturado em 08 dez. 2020]. Disponível em: https://susanalitico.saude.gov.br/extension s/covid-19_html/covid-19_html.html

5. Chan JF, Yuan S, Kok KH, et al. A familial cluster of pneumonia associated with the 2019 novel coronavirus indicating personto-person transmission: a study of a family cluster. Lancet. 2020; 395(10223):514523. $\quad$ https://doi.org/10.1016/S0140$\underline{6736(20) 30154-9}$

6. Shen Z, Lu N, Gao L, et al. Initial chest CT findings in COVID-19: correlation with clinical features. J. Zhejiang Univ. Sci. B. 2020. 21, 668-672.

https://doi.org/10.1631/jzus.B2000133

7. Xie X, Zhong Z, Zhao W, et al. Chest CT for Typical Coronavirus Disease 2019 (COVID-19) Pneumonia: Relationship to Negative RT-PCR Testing. Radiology. 2020; 296(2): E41-E45. https://doi.org/10.1148/radiol.202020034 $\underline{3}$

8. Huang C, Wang Y, Li X, et al. Clinical features of patients infected with 2019 novel coronavirus in Wuhan, China [published correction appears in Lancet. 2020 Jan 30;]. Lancet. 2020;395(10223):497-506.

https://doi.org/10.1016/S0140$\underline{6736(20) 30183-5}$

9. Yang R, Li X, Liu H, et al. (2020) Pontuação de gravidade de TC de tórax: uma ferramenta de imagem para avaliar COVID-19 grave. Radiology: Cardiothoracic Imaging 2020. https://doi.org/10.1148/ryct.202020 $\underline{0047}$

10. Salvatore C, Roberta F, Angela D, et al. Clinical and laboratory data, radiological structured report findings and quantitative evaluation of lung involvement on baseline chest $\mathrm{CT}$ in COVID-19 patients to predict prognosis. Radiol med. 2020. https://doi.org/10.1007/s11547-020$\underline{01293-w}$

11. Meirelles GSP. COVID-19: a brief update for radiologists. Radiologia Brasileira. 2020. 53(5), 320-328.

http://dx.doi.org/10.1590/01003984.2020.0074

12. Niquini RP, Lana RM, Pacheco AG, et al. SRAG por COVID-19 no Brasil: descrição e comparação de características demográficas e comorbidades com SRAG por influenza e com a população geral. Cad. Saúde Pública. 2020; 36(7): e00149420. Epub July 24, 2020. http://dx.doi.org/10.1590/0102$\underline{311 \times 00149420}$

13. Su S, Wong G, Shi W, et al. Epidemiology, Genetic Recombination, and Pathogenesis of Coronaviruses. Trends Microbiol. 2016; $24 \quad$ (6): 490502. https://doi.org/10.1016/j.tim.2016.03 .003

14. Caruso, D., Polici, M., Zerunian, M. et al. Análise quantitativa de TC de tórax na discriminação de pacientes COVID-19 de não COVID-19. Radiol med (2020). https://doi.org/10.1007/s11547-02001291-y

15. Zhou, Z., Guo, D., Li, C. et al. Coronavirus disease 2019: initial chest CT findings. Eur Radiol 30, 4398-4406 (2020).

https://doi.org/10.1007/s00330-02006816-7

16. Li Y, Xia L. Coronavirus Disease 2019 (COVID-19): Role of Chest CT in Diagnosis and Management. American Journal of Roentgenology 2020. https://doi.org/10.2214/AJR.20.22954 